\title{
Sampling of Deterministic Signals and Systems
}

\author{
Holger Boche, Fellow, IEEE, and Ullrich J. Mönich, Student Member, IEEE
}

\begin{abstract}
In this paper, we analyze sampling approximations of stable linear time-invariant systems for input signals in the PaleyWiener space $\mathcal{P} \mathcal{W}_{\pi}^{1}$. The goal is to approximate a transform $T f$ by using only the samples of the signal $f$, which do not necessarily have to be equidistant. We completely characterize the stable linear time-invariant (LTI) systems $T$ and sampling patterns for which the approximation process converges to $T f$ for all signals in $\mathcal{P} \mathcal{W}_{\pi}^{1}$. Furthermore, we prove that for every complete interpolating sequence there exist a stable LTI system and a signal in $\mathcal{P} \mathcal{W}_{\pi}^{1}$ for which the approximation error increases unboundedly as the number of samples that are used for the approximation is increased. This shows that a more flexible choice of the sampling points instead of equidistant sampling, where such a divergence behavior is known to exist, is not sufficient to overcome the divergence. Thus, sampling based signal processing has fundamental limits. The calculations further indicate that oversampling cannot resolve the convergence problems.
\end{abstract}

Index Terms-Approximation, complete interpolating sequence, Paley-Wiener space, sampling series, stable linear time-invariant system.

\section{INTRODUCTION AND NOTATION}

$\mathbf{O}$ NE reason for the success of digital signal processing lies in the fact that certain bandlimited signals are completely determined by their samples and that they can be perfectly reconstructed from these samples. The Shannon sampling theorem [1] was the starting point for many further developments in sampling theory. However, also in other disciplines than signal processing, sampling theory is an appreciated tool. In his "Lectures on Computation" [2], Feynman discusses the theoretical foundations and concepts of classical and quantum computation. One important step in his argumentation is the transition from the continuous-time domain to the discrete-time domain and the Shannon sampling theorem is the theoretical basis, which creates the link between both domains. He discusses the problem of transmitting a function of time and writes in this context: "Consideration of such a problem will bring us on to consider the famous Sampling Theorem, another baby of Claude Shannon" [2, p. 132]. Besides the importance in signal processing and communication theory [1], the reconstruction of bandlimited continuous-time signals from their samples by the

Manuscript received March 09, 2010; revised October 25, 2010; accepted January 04, 2011. Date of publication January 24, 2011; date of current version April 13, 2011. The associate editor coordinating the review of this manuscript and approving it for publication was Prof. Trac D. Tran. This work was supported in part by the German Research Foundation (DFG) under Grant BO 1734/9-1.

The authors were with the Technische Universität Berlin, Heinrich-Hertz-Chair for Mobile Communications, D-10578 Berlin, Germany. They are now with the Technische Universität München, Lehrstuhl für Theoretische Informationstechnik, D-80290 München, Germany (e-mail: boche@tum.de; moenich@tum.de).

Digital Object Identifier 10.1109/TSP.2011.2107903
Shannon sampling series is also essential for other applications and theoretical concepts [3]-[5]. The reconstruction of non-bandlimited signals, which was analyzed, for example, in [6] and [7], will not be considered in this paper. For a general overview of sampling theorems, see [3], [8], and [9].

Often, the interest is not in the signal itself but in some processed version of it. This might be the derivative, the Hilbert transform or the result of any other stable linear system $T$. Thus, the goal is to approximate the desired transform $T f$ of a signal $f$ by an approximation process, which uses only finitely many, not necessarily equidistant samples of the signal $f$. Exactly as in the case of signal reconstruction, the convergence behavior is important for practical applications. In [10], Habib derived a sampling representation for a certain class of stable linear systems acting on signals which are bandlimited in the sense of Zakai [11]. However, important systems like the Hilbert transform and the ideal low-pass filter do not belong to this class.

One possible application for this sampling-based signal processing approach is in sensor networks. In a sensor network, a large number of sensors is used to monitor some physical quantity, e.g., temperature or electric field intensity. This physical quantity varies continuously in space and thus, can be viewed as a signal in space. In general, the sensors are placed non-equidistantly according to the given spacial settings. Thus, in sampling theoretic terminology, the sensors perform a non-equidistant sampling of the signal. At the fusion center, where the data from all sensors is gathered, the task is to reconstruct the signal or, in the case where the signal is further processed, to approximate some transformation of this signal by using only the samples of the signal, which are produced by the sensors.

The sensor network example shows that non-equidistant sampling patterns are important, in particular for practical applications. However, also in other applications, non-equidistant sampling patterns arise quite naturally from the problem setting. For example, it has been shown that bandlimited signals that are bounded and real on the real axis are uniquely determined by their sine wave crossings if the peak value of the signal is smaller than the amplitude of the sine wave [12]-[14]. In this case, the sine wave crossings are all real [15] and the set of sine wave crossings constitutes a possible non-equidistant sampling pattern.

In order to continue, we need some notation and definitions. $L^{p}(\mathbb{R}), 1 \leq p<\infty$, denotes the space of all to the $p$ th power Lebesgue integrable functions on $\mathbb{R}$, with the usual norm $\|\cdot\|_{p}$ and $L^{\infty}(\mathbb{R})$ the space of all functions for which the essential supremum norm $\|\cdot\|_{\infty}$ is finite. $\lambda$ is the Lebesgue measure. Furthermore, $l^{p}, 1 \leq p<\infty$, is the space of all sequences such that the $p$-norm $\|\cdot\|_{l^{p}}$ is finite. $C\left[t_{1}, t_{2}\right]$ denotes the space of all continuous functions on $\left[t_{1}, t_{2}\right]$ and $C_{0}^{\infty}\left[t_{1}, t_{2}\right]$ the space of all infinitely differentiable functions on $\mathbb{R}$ whose support is in $\left[t_{1}, t_{2}\right]$. Further, let $\hat{f}$ denote the Fourier transform of a function 
$f$. For functions in $L^{1}(\mathbb{R})$ the Fourier transform is defined in the classical sense, according to

$$
\hat{f}(\omega)=\int_{-\infty}^{\infty} f(t) \mathrm{e}^{-i \omega t} \mathrm{~d} t,
$$

for functions in $L^{p}(\mathbb{R}), 1<p \leq 2$ as the limit of $\int_{-N}^{N} f(t) \mathrm{e}^{-i \omega t} \mathrm{~d} t$ in $L^{q}(\mathbb{R}), 1 / p+1 / q=1$ and for functions in $L^{p}(\mathbb{R}), p>2$, in the distributional sense. For $\sigma>0$ and $1 \leq p \leq \infty$ we denote by $\mathcal{P W}_{\sigma}^{p}$ the Paley-Wiener space of signals $f$ with a representation $f(z)=1 /(2 \pi) \int_{-\sigma}^{\sigma} g(\omega) \mathrm{e}^{i z \omega} \mathrm{d} \omega, z \in \mathbb{C}$, for some $g \in L^{p}(-\sigma, \sigma)$. If $f \in \mathcal{P W}_{\sigma}^{p}$ then $g(\omega)=\hat{f}(\omega)$. The norm for $\mathcal{P} \mathcal{W}_{\sigma}^{p}, 1 \leq p<\infty$, is given by $\|f\|_{\mathcal{P W}}=$ $\left(1 /(2 \pi) \int_{-\sigma}^{\sigma}|\hat{f}(\omega)|^{p} \mathrm{~d} \omega\right)^{1 / p}$.

Since our analyses involve stable linear time-invariant (LTI) systems, we briefly review some definitions and facts. A linear system $T: \mathcal{P W}_{\pi}^{p} \rightarrow \mathcal{P W}_{\pi}^{p}, 1 \leq p \leq \infty$, is called stable if the operator $T$ is bounded, i.e., if $\|T\|=\sup _{\|f\|_{\mathcal{P W}}^{p} \leq 1}\|T f\|_{\mathcal{P W}_{\pi}^{p}}<\infty$. Furthermore, it is called time-invariant if $(T f(\cdot-a))(t)=(T f)(t-a)$ for all $f \in \mathcal{P} \mathcal{W}_{\pi}^{p}$ and $t, a \in \mathbb{R}$.

Remark 1: Note that our definition of stability is with respect to the $\mathcal{P} \mathcal{W}_{\pi}^{p}$-norm and thus is different from the concept of bounded input-bounded output stability.

For every stable LTI system $T: \mathcal{P} \mathcal{W}_{\pi}^{1} \rightarrow \mathcal{P} \mathcal{W}_{\pi}^{1}$ there exists exactly one function $\hat{h}_{T} \in L^{\infty}[-\pi, \pi]$ such that

$$
(T f)(t)=\frac{1}{2 \pi} \int_{-\pi}^{\pi} \hat{f}(\omega) \hat{h}_{T}(\omega) \mathrm{e}^{i \omega t} \mathrm{~d} \omega
$$

for all $f \in \mathcal{P} \mathcal{W}_{\pi}^{1}$. Conversely, every function $\hat{h}_{T} \in L^{\infty}[-\pi, \pi]$ defines a stable LTI system $T: \mathcal{P} \mathcal{W}_{\pi}^{1} \rightarrow \mathcal{P} \mathcal{W}_{\pi}^{1}$. The operator norm of a stable LTI system $T$ is given by $\|T\|=\|\hat{h}\|_{\infty}$. Furthermore, it can be shown that the representation (1) with $\hat{h}_{T} \in L^{\infty}[-\pi, \pi]$ is also valid for all stable LTI systems $T$ : $\mathcal{P W}_{\pi}^{2} \rightarrow \mathcal{P} \mathcal{W}_{\pi}^{2}$. Therefore, every stable LTI system that maps $\mathcal{P W}_{\pi}^{1}$ in $\mathcal{P} \mathcal{W}_{\pi}^{1}$ maps $\mathcal{P} \mathcal{W}_{\pi}^{2}$ in $\mathcal{P} \mathcal{W}_{\pi}^{2}$, and vice versa. Note that $\hat{h}_{T} \in L^{\infty}[-\pi, \pi] \subset L^{2}[-\pi, \pi]$ and consequently $h_{T} \in \mathcal{P} \mathcal{W}_{\pi}^{2}$.

\section{Motivation AND OutLine}

The discussion in Section I has shown that, in addition to the pure signal reconstruction problem, it is often also important to realize signal processing algorithms. In the sensor network example, for example, the peak value and the dynamics of the physical quantity, i.e., the signal, might be of interest. While the peak value of the signal can be determined in principle from the reconstruction of signal itself, the dynamics requires the computation of the derivative of the signal. Since many signal processing algorithms on bandlimited signals, like the computation of the derivative, are stable LTI systems, the system approximation problem is important. In this paper, we analyze the system approximation problem for stable LTI systems and signals in $\mathcal{P W}_{\pi}^{1}$, i.e., we analyze sampling type approximation processes for the output $T f$ of stable LTI systems $T: \mathcal{P} \mathcal{W}_{\pi}^{1} \rightarrow \mathcal{P W}_{\pi}^{1}$ and signals $f$ in the space $\mathcal{P} \mathcal{W}_{\pi}^{1}$.

The results for the Paley-Wiener space $\mathcal{P} \mathcal{W}_{\pi}^{1}$ are interesting because the space $\mathcal{P} \mathcal{W}_{\pi}^{1}$ is the largest space in the scale of
Paley-Wiener spaces. In particular, it is larger than the intensely studied space $\mathcal{P} \mathcal{W}_{\pi}^{2}$ of bandlimited signals with finite $L^{2}$-norm. Although it is still smaller than the important space of bandlimited signals that are bounded on the real axis, the results for $\mathcal{P W}_{\pi}^{1}$ can be seen as a step from the small space $\mathcal{P} \mathcal{W}_{\pi}^{2}$ towards the larger space of bounded bandlimited signals.

Further, the convergence theory of sampling series for the space $\mathcal{P} \mathcal{W}_{\pi}^{1}$ is closely connected with the mean-square convergence theory of sampling series for bandlimited wide-sense stationary process [16].

In [17], the convergence behavior of approximation processes that use equidistant samples, taken at or above Nyquist rate, was analyzed. Among other things it was shown that there exists a stable LTI system $T_{1}$ and a signal $f_{1} \in \mathcal{P} \mathcal{W}_{\pi}^{1}$ such that

$$
\limsup _{N \rightarrow \infty}\left(\sup _{t \in \mathbb{R}}\left|\left(T_{1} f_{1}\right)(t)-\sum_{k=-N}^{N} f_{1}(k) h_{T_{1}}(t-k)\right|\right)=\infty,
$$

i.e., the peak value of the approximation error grows arbitrarily large. In particular the peak value and the derivative that were discussed above cannot be determined from the samples in this setting.

In this paper, we go one step further and consider more flexible sampling patterns [18]. By using non-equidistant sampling, an additional degree of freedom is created, which may help to improve the convergence behavior. We will analyze whether this additional degree of freedom can be exploited to construct approximation processes that are convergent for all signals in $\mathcal{P} \mathcal{W}_{\pi}^{1}$ and all stable LTI systems. More precisely, we analyze the convergence behavior of the sampling series

$$
\sum_{k=-N}^{N} f\left(t_{k}\right)\left(T \phi_{k}\right)(t)
$$

where $T: \mathcal{P W}_{\pi}^{1} \rightarrow \mathcal{P} \mathcal{W}_{\pi}^{1}$ is a stable LTI system, $\phi_{k} \in$ $\mathcal{P W}_{\pi}^{2}, k \in \mathbb{Z}$, are reconstruction functions and $f$ is a signal in $\mathcal{P} \mathcal{W}_{\pi}^{1}$.

The paper is organized as follows. In Section III, we present the fundamentals of non-equidistant sampling. In particular, we characterize meaningful sampling patterns and discuss the convergence behavior of (2) for signals in $\mathcal{P W} \mathcal{W}_{\pi}^{2}$. Finally, in Section IV, we completely characterize the cases where (2) converges to $T f$ for all $f \in \mathcal{P W}_{\pi}^{1}$ and the cases where (2) diverges for some $f \in \mathcal{P} \mathcal{W}_{\pi}^{1}$. Moreover, we prove the existence of a stable LTI system $T$ and a signal $f \in \mathcal{P W}_{\pi}^{1}$ for which (2) diverges. This shows that there are fundamental limits in sampling based signal processing. In Section V, we discuss the results and present open problems. We conjecture that oversampling itself cannot resolve the convergence problems. Only oversampling in combination with more general sampling functionals may achieve a uniformly convergent approximation process.

\section{FUNDAMENTALS OF NON-EQUIDISTANT SAMPLING}

\section{A. Complete Interpolating Sequences}

In this section, we study some fundamentals of non-equidistant sampling. Throughout the paper, we assume that the sampling points $t_{k}, k \in \mathbb{Z}$, are real and that the sequence 
of sampling points $\left\{t_{k}\right\}_{k \in \mathbb{Z}}$ is ordered strictly increasingly. Moreover, without loss of generality, we assume that $t_{0}=0$ if $0 \in\left\{t_{k}\right\}_{k \in \mathbb{Z}}$.

Although some of the theorems below are also valid for general complex sampling point sequences, we restrict our presentation to sequences of real sampling points that are strictly increasing.

Moreover, for our analysis, we require the sampling points $\left\{t_{k}\right\}_{k \in \mathbb{Z}}$ to fulfill the following minimal property.

Property 1: There are two constants $0<C_{L}, C_{R}<\infty$ such that

$$
C_{L}\|f\|_{\mathcal{P W}_{\pi}^{2}} \leq\left(\sum_{k=-\infty}^{\infty}\left|f\left(t_{k}\right)\right|^{2}\right)^{1 / 2} \leq C_{R}\|f\|_{\mathcal{P} \mathcal{W}_{\pi}^{2}}
$$

for all $f \in \mathcal{P} \mathcal{W}_{\pi}^{2}$ and the left inequality in (3) is no longer true if one or more sampling points are removed.

This minimal property is closely linked with the concept of a complete interpolating sequence. Property 1 is fulfilled if and only if $\left\{t_{k}\right\}_{k \in \mathbb{Z}}$ is a complete interpolating sequence for $\mathcal{P} \mathcal{W}_{\pi}^{2}$. For details about complete interpolating sequences and the norm inequality (3) and further background material, we would like to refer the reader to [19, Lecture 23].

Definition 1: We say that $\left\{t_{k}\right\}_{k \in \mathbb{Z}}$ is a complete interpolating sequence for $\mathcal{P} \mathcal{W}_{\pi}^{2}$ if the interpolation problem $f\left(t_{k}\right)=$ $c_{k}, k \in \mathbb{Z}$, has exactly one solution $f \in \mathcal{P} \mathcal{W}_{\pi}^{2}$ for every sequence $\left\{c_{k}\right\}_{k \in \mathbb{Z}} \in l^{2}$.

Definition 2: A system of vectors $\left\{\phi_{k}\right\}_{k \in \mathbb{Z}}$ in a separable Hilbert space $\mathcal{H}$ is called Riesz basis if $\left\{\phi_{k}\right\}_{k \in \mathbb{Z}}$ is complete in $\mathcal{H}$, and there exist positive constants $A$ and $B$ such that for all $M, N \in \mathbb{N}$ and arbitrary scalars $c_{k}$, we have

$$
A \sum_{k=-M}^{N}\left|c_{k}\right|^{2} \leq\left\|\sum_{k=-M}^{N} c_{k} \phi_{k}\right\|^{2} \leq B \sum_{k=-M}^{N}\left|c_{k}\right|^{2} .
$$

Equation (3) implies that a signal $f \in \mathcal{P W}_{\pi}^{2}$ is completely determined by its values on the sampling grid. It couples the behavior of the continuous-time signal, more precisely its $\mathcal{P} \mathcal{W}_{\pi}^{2}$-norm, with the $l^{2}$-norm of the samples on the grid $\left\{t_{k}\right\}_{k \in \mathbb{Z}}$, i.e., the behavior of the discrete-time signal. This coupling is one reason why complete interpolating sequences are of such high importance.

Another interesting fact [20, p. 143], which establishes a connection between Riesz bases and complete interpolating sequences is the following.

Theorem 1: The system $\left\{\mathrm{e}^{i \omega t_{k}}\right\}_{k \in \mathbb{Z}}$ is a Riesz basis for $L^{2}[-\pi, \pi]$ if and only if $\left\{t_{k}\right\}_{k \in \mathbb{Z}}$ is a complete interpolating sequence for $\mathcal{P} \mathcal{W}_{\pi}^{2}$.

In our preceding discussion, we have seen how important the concept of a complete interpolating sequence is. Therefore, a definition that is less implicit than Definition 1 would be desirable. Lyubarskii and Seip have shown that it is possible to characterize complete interpolating sequences directly in terms of the sequence itself and without using signals $f \in \mathcal{P} \mathcal{W}_{\pi}^{2}$ [21]. We briefly review the definition of a relatively dense sequence and the definition of a function of exponential type, before we state this characterization in Theorem 2.
Definition 3: A sequence $\left\{t_{k}\right\}_{k \in \mathbb{Z}}$ of real numbers is called relatively dense if there exists an $r>0$ such that

$$
\left\{t_{k}\right\}_{k \in \mathbb{Z}} \cap[x-r, x+r] \neq \emptyset \text { for each } x \in \mathbb{R} .
$$

Definition 4: An entire function $f$ is said to be of exponential type if for all $\epsilon>0$ there exists a constant $C_{1}=C_{1}(\epsilon)$ such that

$$
|f(z)| \leq C_{1} \mathrm{e}^{(\sigma+\epsilon)|z|}
$$

for all $z \in \mathbb{C}$. The smallest value $\sigma$ which can be used in (5) is called exponential type of $f$.

For details about entire functions of exponential type, we would like to refer the reader to [19] and [22].

Theorem 2 (Lyubarskii and Seip [21]): A sequence $\left\{t_{k}\right\}_{k \in \mathbb{Z}}$ of real numbers is a complete interpolation sequence for $\mathcal{P W}_{\pi}^{2}$ if and only if the following three conditions are fulfilled:

1) $\left\{t_{k}\right\}_{k \in \mathbb{Z}}$ is uniformly discrete, i.e.,

$$
\inf _{n \neq m}\left|t_{n}-t_{m}\right|>0 \text {. }
$$

2) The limit

$$
\phi(z)=\left(z-t_{0}\right) \lim _{N \rightarrow \infty} \prod_{\substack{t_{k} \mid \leq N \\ k \neq 0}}\left(1-\frac{z}{t_{k}}\right)
$$

exists for all finite $z \in \mathbb{C}$ and represents an entire function of exponential type $\pi$.

3) There exists a constant $C_{2}$ and a relatively dense subsequence $\left\{t_{l_{k}}\right\}_{k \in \mathbb{Z}}$, such that for all finite intervals $I \subset \mathbb{R}$

$$
\left(\frac{1}{|I|} \sum_{k \in \mathbb{Z} \cap I}\left|\phi^{\prime}\left(t_{l_{k}}\right)\right|^{2}\right)\left(\frac{1}{|I|} \sum_{k \in \mathbb{Z} \cap I} \frac{1}{\left|\phi^{\prime}\left(t_{l_{k}}\right)\right|^{2}}\right)<C_{2} \text {. }
$$

Aside from the necessary and sufficient condition in Theorem 2, there are other conditions for a sequence to be a complete interpolating sequence for $\mathcal{P} \mathcal{W}_{\pi}^{2}$ that are simpler but only sufficient. One example is Kadec's 1/4-Theorem [20, p. 42], which answers the question when the set $\left\{\mathrm{e}^{i \omega t_{k}}\right\}_{k \in \mathbb{Z}}$ forms a Riesz basis for $L^{2}[-\pi, \pi]$.

Theorem 3 (Kadec's 1/4-Theorem): If $\left\{t_{k}\right\}_{k \in \mathbb{Z}}$ is a sequence of real numbers for which

$$
\left|t_{k}-k\right| \leq \bar{\delta}<\frac{1}{4}, \quad k \in \mathbb{Z},
$$

then $\left\{\mathrm{e}^{i \omega t_{k}}\right\}_{k \in \mathbb{Z}}$ forms a Riesz basis for $L^{2}[-\pi, \pi]$.

Since we assume that the sequence $\left\{t_{k}\right\}_{k \in \mathbb{Z}}$ is a complete interpolating sequence for $\mathcal{P} \mathcal{W}_{\pi}^{2}$, it follows by definition, that for each $k \in \mathbb{Z}$ there is exactly one function $\phi_{k} \in \mathcal{P W}_{\pi}^{2}$ that solves the interpolation problem

$$
\phi_{k}\left(t_{l}\right)= \begin{cases}1, & l=k \\ 0, & l \neq k\end{cases}
$$

According to (3), the norm of $\phi_{k}$ satisfies $\left\|\phi_{k}\right\|_{\mathcal{P W}} \leq 1 / C_{L}$, where $C_{L}$ is the same constant as in (3). On the other hand, if the sequence of real and increasingly ordered sampling points $\left\{t_{k}\right\}_{k \in \mathbb{Z}}$ is a complete interpolating sequence for $\mathcal{P} \mathcal{W}_{\pi}^{2}$, then the product in (6) converges uniformly on $|z|<L$ for all $L<\infty$ 
and $\phi$ as defined by (6) is an entire function of exponential type $\pi[19]$. As a consequence,

$$
\phi_{k}(t)=\frac{\phi(t)}{\phi^{\prime}\left(t_{k}\right)\left(t-t_{k}\right)}
$$

is the unique function in $\mathcal{P} \mathcal{W}_{\pi}^{2}$ that solves the interpolation problem (7).

Example 1: The following simple example illustrates the aforementioned properties. In the case of equidistant samples $t_{k}=k, k \in \mathbb{Z}$, the theory reduces to the well known Shannon sampling series with sinc-kernel. We have

$$
\phi(t)=\lim _{N \rightarrow \infty} t \prod_{0<|k| \leq N}\left(1-\frac{t}{k}\right)=\sin (\pi t) .
$$

Furthermore, $\phi^{\prime}\left(t_{k}\right)=\pi \cos \left(\pi t_{k}\right)=\pi(-1)^{k}$, and it turns out that

$$
\phi_{k}(t)=\frac{\phi(t)}{\phi^{\prime}\left(t_{k}\right)\left(t-t_{k}\right)}=\frac{(-1)^{k} \sin (\pi t)}{\pi\left(t-t_{k}\right)}=\frac{\sin (\pi(t-k))}{\pi(t-k)}
$$

is the well-known sinc-kernel. Of course, $\phi_{k}$ has the interpolation property (7) and the interpolation problem $f\left(t_{k}\right)=f(k)=$ $c_{k}, k \in \mathbb{Z}$, has exactly one solution $f \in \mathcal{P} \mathcal{W}_{\pi}^{2}$ for every sequence $\left\{c_{k}\right\}_{k \in \mathbb{Z}} \in l^{2}$. This solution is given by the Shannon sampling series

$$
f(t)=\sum_{k=-\infty}^{\infty} c_{k} \frac{\sin (\pi(t-k))}{\pi(t-k)} .
$$

In the case of equidistant sampling at Nyquist rate, the energy of the discrete-time signal equals the energy of the continuoustime signal

$$
\|f\|_{\mathcal{P} \mathcal{W}_{\pi}^{2}}^{2}=\sum_{k=-\infty}^{\infty}|f(k)|^{2} .
$$

If the sampling pattern is relaxed from the constraint of being equidistant, equality (9) cannot be supposed to be true any longer, but, as we have seen, we have at least the norm equivalence (3) if the sequence of sampling points $\left\{t_{k}\right\}_{k \in \mathbb{Z}}$ is a complete interpolating sequence for $\mathcal{P W}_{\pi}^{2}$.

The norm equivalence (3) is very useful for the convergence analysis of the sampling series

$$
\sum_{k=-\infty}^{\infty} f\left(t_{k}\right) \phi_{k} .
$$

For $f \in \mathcal{P W}_{\pi}^{2}$, we have

$$
\begin{aligned}
\| f & -\sum_{k=-N}^{N} f\left(t_{k}\right) \phi_{k} \|_{\mathcal{P} \mathcal{W}_{\pi}^{2}} \\
& \leq \frac{1}{C_{L}}\left(\sum_{l=-\infty}^{\infty}\left|f\left(t_{l}\right)-\sum_{k=-N}^{N} f\left(t_{k}\right) \phi_{k}\left(t_{l}\right)\right|^{2}\right)^{1 / 2} \\
& =\frac{1}{C_{L}}\left(\sum_{|k|>N}\left|f\left(t_{k}\right)\right|^{2}\right)^{1 / 2}
\end{aligned}
$$

where we used (3) in the first inequality and (7) in the last equality. Since

$$
\left(\sum_{k=-\infty}^{\infty}\left|f\left(t_{k}\right)\right|^{2}\right)^{1 / 2} \leq C_{R}\|f\|_{\mathcal{P} \mathcal{W}_{\pi}^{2}}^{2}<\infty
$$

according to (3), it follows that

$$
\lim _{N \rightarrow \infty}\left(\sum_{|k|>N}\left|f\left(t_{k}\right)\right|^{2}\right)^{1 / 2}=0
$$

and, consequently,

$$
\lim _{N \rightarrow \infty}\left\|f-\sum_{k=-N}^{N} f\left(t_{k}\right) \phi_{k}\right\|_{\mathcal{P W}_{\pi}^{2}}=0
$$

for all $f \in \mathcal{P} \mathcal{W}_{\pi}^{2}$. Moreover, since $\|f\|_{\infty} \leq\|f\|_{\mathcal{P W}_{\pi}^{2}}$, this implies that

$$
\lim _{N \rightarrow \infty}\left\|f-\sum_{k=-N}^{N} f\left(t_{k}\right) \phi_{k}\right\|_{\infty}=0
$$

for all signals $f \in \mathcal{P} \mathcal{W}_{\pi}^{2}$.

Further, if the sequence of sampling points $\left\{t_{k}\right\}_{k \in \mathbb{Z}}$ is a complete interpolating sequence for $\mathcal{P} \mathcal{W}_{\pi}^{2}$, sampling based signal processing is possible for $f \in \mathcal{P W}_{\pi}^{2}$, because for all stable LTI systems $T: \mathcal{P W}_{\pi}^{2} \rightarrow \mathcal{P W}_{\pi}^{2}$ and $f \in \mathcal{P W}_{\pi}^{2}$ we have

$$
\begin{gathered}
\left\|T f-\sum_{k=-N}^{N} f\left(t_{k}\right) T \phi_{k}\right\|_{\mathcal{P W}_{\pi}^{2}} \\
=\left\|T\left(f-\left(\sum_{k=-N}^{N} f\left(t_{k}\right) \phi_{k}\right)\right)\right\|_{\mathcal{P W}_{\pi}^{2}} \\
\leq\|T\|\left\|f-\sum_{k=-N}^{N} f\left(t_{k}\right) \phi_{k}\right\|_{\mathcal{P W}_{\pi}^{2}}
\end{gathered}
$$

and the right-hand side of (12) converges to zero according to (11). Thus, we have

$$
\lim _{N \rightarrow \infty}\left\|T f-\sum_{k=-N}^{N} f\left(t_{k}\right) T \phi_{k}\right\|_{\mathcal{P W}_{\pi}^{2}}=0
$$

and, due to $\|f\|_{\infty} \leq\|f\|_{\mathcal{P} \mathcal{W}_{\pi}^{2}}$, that

$$
\lim _{N \rightarrow \infty}\left\|T f-\sum_{k=-N}^{N} f\left(t_{k}\right) T \phi_{k}\right\|_{\infty}=0
$$

for all signals $f \in \mathcal{P} \mathcal{W}_{\pi}^{2}$.

Equations (13) and (14) show that, for $f \in \mathcal{P W}_{\pi}^{2}$, the finite sampling series with transformed kernel (2) converges to the transformed signal $T f$ in the $\mathcal{P} \mathcal{W}_{\pi}^{2}$-norm and in the maximum-norm. This means, the transformed signal $T f$ can be arbitrarily well approximated by the finite sampling series (2). From (10) and (12), we can again see the significance of complete interpolating sequences, because the convergence behavior and in particular the convergence speed of (2) is determined by the 
samples of the signal $f \in \mathcal{P} \mathcal{W}_{\pi}^{2}$. Equation (2) can be regarded as a model for signal processing on a discrete set.

\section{B. Zeros of Sine-Type Functions}

In the previous section, we have seen that (2) converges for all signals in $\mathcal{P W}_{\pi}^{2}$ and all sampling patterns that are complete interpolating sequences for $\mathcal{P} \mathcal{W}_{\pi}^{2}$. Before we tackle the problem of analyzing the convergence behavior of (2) for signals in $\mathcal{P} \mathcal{W}_{\pi}^{1}$, we review some results on the convergence behavior of (2) for signals in $\mathcal{P} \mathcal{W}_{\pi}^{1}$ and the special case where $T$ is the identity operator.

Definition 5: An entire function $f$ of exponential type $\pi$ is said to be of sine type if the zeros of $f$ are separated and simple, and there exist positive constants $A, B$ and $H$ such that $A \mathrm{e}^{\pi|y|} \leq|f(x+i y)| \leq B \mathrm{e}^{\pi|y|}$ whenever $x$ and $y$ are real and $|y| \geq H$.

Example 2: $\sin (\pi z)$ is a function of sine type, and its zeros are $t_{k}=k, k \in \mathbb{Z}$.

Example 3: For signals $f \in \mathcal{P} \mathcal{W}_{\pi}^{1}$ that are real on the real axis, the function $\psi_{f}(z)=f(z)-A \sin (\pi z)$ is a sine-type function if $A>\|f\|_{\infty}$.

For further information about sine-type functions, we would like to refer the reader to [19] and [23].

There is an important connection between the set of zeros $\left\{t_{k}\right\}_{k \in \mathbb{Z}}$ of a function of sine type, the basis properties of the system of exponentials $\left\{\mathrm{e}^{i \omega t_{k}}\right\}_{k \in \mathbb{Z}}$ and complete interpolating sequences.

Lemma 1: If $\left\{t_{k}\right\}_{k \in \mathbb{Z}}$ is the set of zeros of a function of sine type, then the system $\left\{\mathrm{e}^{i \omega t_{k}}\right\}_{k \in \mathbb{Z}}$ is a Riesz basis for $L^{2}[-\pi, \pi]$ and $\left\{t_{k}\right\}_{k \in \mathbb{Z}}$ is a complete interpolating sequence for $\mathcal{P} \mathcal{W}_{\pi}^{2}$.

Proof: This lemma is a simple consequence of Theorems 9 and 10 in [23, pp. 143 and 144].

Lemma 1 implies that if $\phi$ is a function of sine type with zeros $\left\{t_{k}\right\}_{k \in \mathbb{Z}}$, then $\left\{\phi_{k}\right\}_{k \in \mathbb{Z}}$, where $\phi_{k}$ is given by (8), is a Riesz basis for $\mathcal{P W}_{\pi}^{2}[19$, p. 169, Theorem 1].

For sampling patterns that are made of the zeros of sine-type functions, we have a good local convergence behavior of

$$
\sum_{k=-N}^{N} f\left(t_{k}\right) \phi_{k}(t)
$$

for all $f \in \mathcal{P} \mathcal{W}_{\pi}^{1}$. In [24], the uniform convergence on compact subsets of $\mathbb{R}$ of the sampling series (15) was proved for signals in $\mathcal{P} \mathcal{W}_{\pi}^{1}$ and sampling patterns that are made of the zeros of sine-type functions.

Theorem 4: Let $\phi$ be a function of sine type, whose zeros $\left\{t_{k}\right\}_{k \in \mathbb{Z}}$ are all real and ordered increasingly and let $\phi_{k}, k \in \mathbb{Z}$, be the corresponding reconstruction functions as defined in (8). Further, let $0<\zeta<\infty$. Then, we have

$$
\lim _{N \rightarrow \infty} \max _{t \in[-\zeta, \zeta]}\left|f(t)-\sum_{k=-N}^{N} f\left(t_{k}\right) \phi_{k}(t)\right|=0
$$

for all $f \in \mathcal{P} \mathcal{W}_{\pi}^{1}$.
Remark 2: Since $\left\{t_{k}\right\}_{k \in \mathbb{Z}}$ is a complete interpolating sequence for $\mathcal{P} \mathcal{W}_{\pi}^{2}$, the function $\phi$, defined by (6) and as a consequence the functions $\phi_{k}, k \in \mathbb{Z}$, defined by (8), are uniquely determined. Hence, there is no degree of freedom in the choice of the functions $\phi_{k}$.

Note that Theorem 4 makes no statement about the global convergence behavior of the sampling series. Although $\zeta>0$ can be arbitrary, it has to be fixed. The global convergence behavior is more problematic. In [25], it was shown that for all members of a certain subclass of sampling patterns the peak value of (15) diverges for some signal $f_{1} \in \mathcal{P} \mathcal{W}_{\pi}^{1}$. This subclass is determined by the sine-type functions that have a representation as Fourier-Stieltjes integral in the form

$$
\phi(t)=\frac{1}{2 \pi} \int_{-\pi}^{\pi} \mathrm{e}^{i \omega t} \mathrm{~d} \mu(\omega)
$$

where $\mu(\omega)$ is a real function of bounded variation on the interval $[-\pi, \pi]$ that has a jump discontinuity at each endpoint.

Theorem 5: Let $\phi$ be a function of sine type that has the representation (16) and whose zeros $\left\{t_{k}\right\}_{k \in \mathbb{Z}}$ are all real and ordered increasingly. Let $\phi_{k}, k \in \mathbb{Z}$, be the corresponding reconstruction functions as defined in (8). Then there exists a signal $f_{1} \in \mathcal{P} \mathcal{W}_{\pi}^{1}$ such that

$$
\limsup _{N \rightarrow \infty} \max _{t \in \mathbb{R}}\left|f_{1}(t)-\sum_{k=-N}^{N} f_{1}\left(t_{k}\right) \phi_{k}(t)\right|=\infty .
$$

Remark 3: In particular, $\sin (\pi t)$ is a sine-type function that has the representation (16). Hence, Theorem 5 implies that

$$
\limsup _{N \rightarrow \infty} \max _{t \in \mathbb{R}}\left|f_{1}(t)-\sum_{k=-N}^{N} f_{1}(k) \frac{\sin (\pi(t-k))}{\pi(t-k)}\right|=\infty,
$$

i.e., the peak approximation error of the Shannon sampling series with equidistant sampling points diverges for some signal $f_{1} \in \mathcal{P} \mathcal{W}_{\pi}^{1}$.

Theorem 5 was one of the motivations why we conjectured in [26] that the divergence occurs even for larger classes of sampling patterns.

Conjecture 1: Let $\left\{t_{k}\right\}_{k \in \mathbb{Z}} \subset \mathbb{R}$ be complete interpolating sequences for $\mathcal{P W}_{\pi}^{2}$ and $\phi_{k}, k \in \mathbb{Z}$, the corresponding reconstruction functions as defined in (8). Then, there exists a signal $f_{1} \in \mathcal{P} \mathcal{W}_{\pi}^{1}$ such that

$$
\limsup _{N \rightarrow \infty} \max _{t \in \mathbb{R}}\left|f_{1}(t)-\sum_{k=-N}^{N} f_{1}\left(t_{k}\right) \phi_{k}(t)\right|=\infty .
$$

In Conjecture 1, we make a statement about the convergence behavior of the sampling series (2) for the case where $T$ is the identity operator, or in other words, for the case where no system is present. In Theorem 7, we will analyze the sampling series (2), i.e., the case where a stable LTI system $T$ is present and show that for every complete interpolating sequence there exist a stable LTI system $T$ and a signal in $\mathcal{P} \mathcal{W}_{\pi}^{1}$, so that we have 
divergence. Hence, the modification of Conjecture 1, where we additionally apply a stable LTI system, is true.

\section{NON-EQUIDISTANT SAMPLING FOR $\mathcal{P} \mathcal{W}_{\pi}^{1}$}

In Section III, we have seen that, given any sampling point sequence $\left\{t_{k}\right\}_{k \in \mathbb{Z}} \subset \mathbb{R}$ that is a complete interpolating sequence for $\mathcal{P} \mathcal{W}_{\pi}^{2}$, the sampling series (2) is uniformly convergent for all $f \in \mathcal{P}^{2} \mathcal{W}_{\pi}^{2}$ and all stable LTI systems $T: \mathcal{P} \mathcal{W}_{\pi}^{2} \rightarrow \mathcal{P} \mathcal{W}_{\pi}^{2}$. However, for $f \in \mathcal{P} \mathcal{W}_{\pi}^{1}$ the convergence behavior is essentially more intricate, because (3) cannot be used anymore.

In this section, we analyze the convergence behavior of the sampling series (2) for signals $f \in \mathcal{P} \mathcal{W}_{\pi}^{1}$. Theorem 6 gives a necessary and sufficient condition for the convergence of (2) to the transformed signal $T f$ for all $f \in \mathcal{P} \mathcal{W}_{\pi}^{1}$. Later, in the proof of Theorem 7, we will use Theorem 6 to show that for every sampling pattern $\left\{t_{k}\right\}_{k \in \mathbb{Z}} \subset \mathbb{R}$ that is a complete interpolating sequence for $\mathcal{P} \mathcal{W}_{\pi}^{2}$ there exists a stable LTI system $T$ : $\mathcal{P} \mathcal{W}_{\pi}^{1} \rightarrow \mathcal{P} \mathcal{W}_{\pi}^{1}$ and a signal $f \in \mathcal{P} \mathcal{W}_{\pi}^{1}$ such that (2) diverges for a fixed $t \in \mathbb{R}$. Thus, it will turn out that the additional degree of freedom in the choice of the sampling points, compared to equidistant sampling, cannot prevent the approximation process (2) to diverge for some stable LTI system $T: \mathcal{P} \mathcal{W}_{\pi}^{1} \rightarrow \mathcal{P} \mathcal{W}_{\pi}^{1}$ and some signal $f \in \mathcal{P} \mathcal{W}_{\pi}^{1}$.

Theorem 6: Let $\left\{t_{k}\right\}_{k \in \mathbb{Z}} \subset \mathbb{R}$ be a complete interpolating sequence for $\mathcal{P} \mathcal{W}_{\pi}^{2}, \phi_{k}, k \in \mathbb{Z}$, the corresponding reconstruction functions as defined in (8), T a stable LTI system and $\mathcal{I}$ a closed subset of $\mathbb{R}$. For all $f \in \mathcal{P} \mathcal{W}_{\pi}^{1}$ we have

$$
\lim _{N \rightarrow \infty} \max _{t \in \mathcal{I}}\left|(T f)(t)-\sum_{k=-N}^{N} f\left(t_{k}\right)\left(T \phi_{k}\right)(t)\right|=0
$$

if and only if there exists a constant $C_{3}<\infty$ such that

$$
\max _{t \in \mathcal{I}} \max _{|\omega| \leq \pi}\left|\sum_{k=-N}^{N} \mathrm{e}^{i \omega t_{k}}\left(T \phi_{k}\right)(t)\right| \leq C_{3}
$$

for all $N \in \mathbb{N}$. If (17) is not fulfilled, then there exists a signal $f_{1} \in \mathcal{P} \mathcal{W}_{\pi}^{1}$ such that

$$
\limsup _{N \rightarrow \infty} \max _{t \in \mathcal{I}}\left|\left(T f_{1}\right)(t)-\sum_{k=-N}^{N} f_{1}\left(t_{k}\right)\left(T \phi_{k}\right)(t)\right|=\infty \text {. }
$$

Remark 4: Note that due to the generality of the set $\mathcal{I}$, Theorem 6 comprises several results for different types of convergence. If the set $\mathcal{I}$ contains only a single point then the theorem makes a statement about the pointwise convergence and if $\mathcal{I}=\mathbb{R}$ then Theorem 6 deals with uniform convergence. Uniform convergence on all of $\mathbb{R}$ is important whenever the peak value of the reconstruction has to be controlled over the whole real axis.

Remark 5: Condition (17) has an interesting interpretation in terms of test signals. The exponential function $f_{\omega}^{\text {test }}(t)=\mathrm{e}^{i \omega t}$ can be thought of as a test signal, where the parameter $\omega$ ranges from $-\pi$ to $\pi$. If

$$
\max _{t \in \mathcal{I}} \sum_{k=-N}^{N} f_{\omega}^{\text {test }}\left(t_{k}\right)\left(T \phi_{k}\right)(t)
$$

is uniformly bounded with respect $\omega \in[-\pi, \pi]$ and $N \in \mathbb{N}$ then the sampling series (2) converges for all $f \in \mathcal{P} \mathcal{W}_{\pi}^{1}$. Although the test signals do not belong to the signal space $\mathcal{P} \mathcal{W}_{\pi}^{1}$, they have an appealingly simple structure. They are just scaled versions of one basic function $\mathrm{e}^{i t}$.

For the proof of Theorem 6, we need Lemma 2, the proof of which is given in Appendix A.

Lemma 2: Let $\left\{t_{k}\right\}_{k \in \mathbb{Z}} \subset \mathbb{R}$ be a complete interpolating sequence for $\mathcal{P} \mathcal{W}_{\pi}^{2}, \phi_{k}, k \in \mathbb{Z}$, the corresponding reconstruction functions as defined in (8), T a stable LTI system, $t \in \mathbb{R}$ and $N \in \mathbb{N}$. Then, we have

$$
\begin{aligned}
\sup _{\|f\|_{\mathcal{P} \mathcal{W}_{\pi}^{1} \leq 1}}\left|\sum_{k=-N}^{N} f\left(t_{k}\right)\left(T \phi_{k}\right)(t)\right| \\
=\max _{|\omega| \leq \pi}\left|\sum_{k=-N}^{N} \mathrm{e}^{i \omega t_{k}}\left(T \phi_{k}\right)(t)\right| .
\end{aligned}
$$

Proof of Theorem 6: The proof consists of two parts. The first part proves the " $\Leftarrow$ " direction of the "if and only if" assertion and the second part the second assertion of the theorem. Since the second assertion implies the " $\Rightarrow$ " direction of the "if and only if" assertion, the whole theorem is proved.

For the proof, we introduce the abbreviation

$$
\left(T_{N} f\right)(t)=\sum_{k=-N}^{N} f\left(t_{k}\right)\left(T \phi_{k}\right)(t)
$$

First part, " $\Leftarrow$ ": Let (17) be fulfilled and $f \in \mathcal{P} \mathcal{W}_{\pi}^{1}$ be arbitrary but fixed. For each $\epsilon>0$ there exists a $g \in \mathcal{P} \mathcal{W}_{\pi}^{2}$ such that $\|f-g\|_{\mathcal{P} \mathcal{W}_{\tau}^{1}}<\epsilon$ and consequently $\max _{t \in \mathcal{I}} \mid(T f)(t)-$ $(T g)(t) \mid<\epsilon\|T\|$. Furthermore,

$$
\begin{aligned}
& \max _{t \in \mathcal{I}}\left|\left(T_{N}(g-f)\right)(t)\right| \\
& =\max _{t \in \mathcal{I}}\left|\frac{1}{2 \pi} \int_{-\pi}^{\pi}(\hat{g}(\omega)-\hat{f}(\omega)) \sum_{k=-N}^{N} \mathrm{e}^{i \omega t_{k}}\left(T \phi_{k}\right)(t) \mathrm{d} \omega\right| \\
& \quad \leq \max _{t \in \mathcal{I}} \frac{1}{2 \pi} \int_{-\pi}^{\pi}|\hat{g}(\omega)-\hat{f}(\omega)|\left|\sum_{k=-N}^{N} \mathrm{e}^{i \omega t_{k}}\left(T \phi_{k}\right)(t)\right| \mathrm{d} \omega \\
& \quad \leq \max _{t \in \mathcal{I}} \max _{|\omega| \leq \pi}\left|\sum_{k=-N}^{N} \mathrm{e}^{i \omega t_{k}}\left(T \phi_{k}\right)(t)\right|\|g-f\|_{\mathcal{P} \mathcal{W}_{\pi}^{1}} \leq C_{3} \epsilon
\end{aligned}
$$

where we used the assumption (17) in the last inequality. Moreover, because of (14), there exists a $N_{0}=N_{0}(\epsilon)$ such that $\max _{t \in \mathcal{I}}\left|(T g)(t)-\left(T_{N} g\right)(t)\right|<\epsilon$ for all $N \geq N_{0}$. Since

$$
\begin{aligned}
& \max _{t \in \mathcal{I}}\left|(T f)(t)-\left(T_{N} f\right)(t)\right| \\
& =\max _{t \in \mathcal{I}} \mid(T f)(t)-(T g)(t)+(T g)(t) \\
& \quad-\left(T_{N} g\right)(t)+\left(T_{N}(g-f)\right)(t) \mid \\
& \leq \max _{t \in \mathcal{I}}|(T f)(t)-(T g)(t)|+\max _{t \in \mathcal{I}}\left|(T g)(t)-\left(T_{N} g\right)(t)\right| \\
& \quad+\max _{t \in \mathcal{I}}\left|\left(T_{N}(g-f)\right)(t)\right|,
\end{aligned}
$$


we obtain $\max _{t \in \mathcal{I}}\left|(T f)(t)-\left(T_{N} f\right)(t)\right|<\left(1+\|T\|+C_{3}\right) \epsilon$ for all $N \geq N_{0}$, and the proof of the first part is complete because $\epsilon$ was arbitrary.

Second part: If (17) is not fulfilled, we have

$$
\begin{aligned}
\limsup _{N \rightarrow \infty} \max _{t \in \mathcal{I}} \max _{|\omega| \leq \pi} \mid & \sum_{k=-N}^{N} \mathrm{e}^{i \omega t_{k}}\left(T \phi_{k}\right)(t) \mid \\
& =\limsup _{N \rightarrow \infty} \max _{t \in \mathcal{I}} \sup _{\|f\|_{\mathcal{P W}}^{1} \leq 1}\left|\left(T_{N} f\right)(t)\right|=\infty
\end{aligned}
$$

because

$$
\sup _{\|f\|_{\mathcal{P} \mathcal{W}_{\pi}^{1}} \leq 1}\left|\left(T_{N} f\right)(t)\right|=\max _{|\omega| \leq \pi}\left|\sum_{k=-N}^{N} \mathrm{e}^{i \omega t_{k}}\left(T \phi_{k}\right)(t)\right|
$$

by Lemma 2. Thus, the Banach-Steinhaus theorem [27, p. 98] implies that there exists a signal $f_{1} \in \mathcal{P} \mathcal{W}_{\pi}^{1}$ such that

$$
\limsup _{N \rightarrow \infty} \max _{t \in \mathcal{I}}\left|\left(T_{N} f_{1}\right)(t)\right|=\infty \text {. }
$$

Since $\max _{t \in \mathcal{I}}\left|\left(T f_{1}\right)(t)\right| \leq\left\|T f_{1}\right\|_{\infty}<\infty$, we have (18) and the proof is complete.

In Theorem 6, we have completely characterized the cases where (2) converges to the signal $T f$ for all $f \in \mathcal{P} \mathcal{W}_{\pi}^{1}$. We will now show that, for every sampling pattern $\left\{t_{k}\right\}_{k \in \mathbb{Z}} \subset \mathbb{R}$ that is a complete interpolating sequence for $\mathcal{P W}_{\pi}^{2}$, there really exists a stable LTI system $T$ such that (2) diverges for some $f \in \mathcal{P W}_{\pi}^{1}$.

Theorem 7: Let $\left\{t_{k}\right\}_{k \in \mathbb{Z}} \subset \mathbb{R}$ be a complete interpolating sequence for $\mathcal{P W}_{\pi}^{2}$ and $\phi_{k}, k \in \mathbb{Z}$, the corresponding reconstruction functions as defined in (8). Then, for all $t \in \mathbb{R}$, there exists a stable LTI system $T_{1}$ with continuous $\hat{h}_{T_{1}}$ and a signal $f_{1} \in \mathcal{P} \mathcal{W}_{\pi}^{1}$ such that

$$
\limsup _{N \rightarrow \infty}\left|\left(T_{1} f_{1}\right)(t)-\sum_{k=-N}^{N} f_{1}\left(t_{k}\right)\left(T_{1} \phi_{k}\right)(t)\right|=\infty .
$$

Theorem 7 shows that, in general, it is not possible to approximate the output of a stable LTI system $T f$ by the sampling series (2), because we can find a signal $f \in \mathcal{P} \mathcal{W}_{\pi}^{1}$ and a stable LTI system $T$ such that (2) diverges for some arbitrary given $t \in \mathbb{R}$.

In order to prove Theorem 7, we need Lemma 3, which is an application of the proposition in [28, p. 57] by Szarek and Lemma 4, which is a consequence of Lusin's theorem.

The divergence results in Section II for equidistant sampling series were based on the analysis of the inverse Fourier integral of a Fourier series. This can be seen from the following identity:

$$
\begin{aligned}
& \sum_{k=-N}^{N} f(k) \frac{\sin (\pi(t-k))}{\pi(t-k)} \\
& =\frac{1}{2 \pi} \int_{-\pi}^{\pi}\left(\sum_{k=-N}^{N} f(k) \mathrm{e}^{-i \omega k}\right) \mathrm{e}^{i \omega t} \mathrm{~d} \omega
\end{aligned}
$$

where we identify the sum inside the parenthesis on the righthand side of (19) as the partial sum of the Fourier series of the $2 \pi$-periodic extension of $\hat{f}$. A well-known result about Fourier series is the du Bois-Reymond theorem [29], which states the existence of a continuous functions whose Fourier series diverges at a some point. This result is equivalent to the divergence of the $L^{1}[-\pi, \pi]$-norm of the Dirichlet kernel

$$
\frac{1}{2 \pi} \int_{-\pi}^{\pi}\left|\frac{\sin \left(\left(N+\frac{1}{2}\right) \omega\right)}{\sin \left(\frac{\omega}{2}\right)}\right| \mathrm{d} \omega=\frac{1}{2 \pi} \int_{-\pi}^{\pi}\left|\sum_{k=-N}^{N} \mathrm{e}^{i k \omega}\right| \mathrm{d} \omega
$$

as $N$ goes to infinity. In order to treat the non-equidistant sampling series in Theorem 7 we use a proposition by Szarek which shows that the divergence is not restricted to Fourier series but equally applies to bounded biorthogonal systems.

Proposition 1 (Szarek): Let $(S, \mathcal{B}, m)$ a probability space and $\left(f_{i}, g_{i}\right)_{i=1}^{N}$ a biorthogonal sequence of measurable functions on $S$ (i.e., $\int_{S} f_{i} g_{j} \mathrm{~d} m=\delta_{i j}$ ) such that

1) $\left\|g_{i}\right\|_{\infty} \leq 1$ for $i=1,2, \ldots, N$;

2) $\int_{S}\left|\sum_{i=1}^{N} s_{i} f_{i}\right|^{2} \mathrm{~d} m \leq C \sum_{k=1}^{N}\left|s_{i}\right|^{2}$ for some $C>0$ and for all sequences of scalars $s_{1}, \ldots, s_{N}$ (and as a consequence, $\int_{S}\left|\sum_{i=1}^{N} t_{i} g_{i}\right|^{2} \mathrm{~d} m \geq C^{-1} \sum_{i=1}^{N}\left|t_{i}\right|^{2}$ for all scalars $\left.t_{1}, \ldots, t_{N}\right)$.

Then there exists $C^{\prime}>0$, depending only on $C$, such that

$$
\max _{1 \leq k \leq N} \int_{S} \int_{S}\left|\sum_{i=1}^{k} g_{i}(t) f_{i}(s)\right| m(d t) m(d s) \geq C^{\prime} \log N .
$$

The next Lemma is a simple corollary of Szarek's proposition.

Lemma 3: Let $\left\{t_{k}\right\}_{k \in \mathbb{Z}} \subset \mathbb{R}$ be a complete interpolating sequence for $\mathcal{P W}_{\pi}^{2}$ and $\phi_{k}, k \in \mathbb{Z}$ the corresponding reconstruction functions as defined in (8). Then, there exists a constant $C_{4}>0$ such that

$$
\begin{array}{r}
\max _{0 \leq M \leq N}\left(\frac{1}{2 \pi}\right)^{2} \int_{-\pi}^{\pi} \int_{-\pi}^{\pi}\left|\sum_{k=-M}^{M} \mathrm{e}^{i \omega t_{k}} \hat{\phi}_{k}(\eta)\right| \mathrm{d} \omega \mathrm{d} \eta \\
\geq C_{4} \log (N)-\frac{1}{C_{L}}
\end{array}
$$

for all $N \in \mathbb{N} . C_{L}$ is the same constant as in (3).

Proof: The proof of Lemma 3 is given in Appendix B.

Remark 6: For the special case of equidistant sampling, we have $t_{k}=k$ and $\hat{\phi}_{k}(\eta)=\mathrm{e}^{-i \eta k}$ and the left-hand side of (21) in Lemma 3 simplifies to

$$
\max _{0 \leq M \leq N}\left(\frac{1}{2 \pi}\right)^{2} \int_{-\pi}^{\pi} \int_{-\pi}^{\pi}\left|\sum_{k=-M}^{M} \mathrm{e}^{i \omega k} \mathrm{e}^{-i \eta k}\right| \mathrm{d} \omega \mathrm{d} \eta,
$$

and using the $2 \pi$-periodicity of the Dirichlet kernel, we obtain

$$
\max _{0 \leq M \leq N} \frac{1}{2 \pi} \int_{-\pi}^{\pi}\left|\sum_{k=-M}^{M} \mathrm{e}^{i k \omega}\right| \mathrm{d} \omega \geq C_{4} \log (N)-\frac{1}{C_{L}} .
$$

If we compare (20) and (22) we see how Szarek's result is related to the divergence of Fourier series and the divergence of the $L^{1}[-\pi, \pi]$-norm of the Dirichlet kernel. Szarek's proposition is more general, because it generalizes the divergence result from orthogonal systems to general bounded biorthogonal systems. However, to achieve this generality, the additional maximum in front of (22) is necessary. 
Lemma 4: For every function $g \in L^{\infty}[-\pi, \pi]$ and every $\epsilon>0$ there exists a function $f_{\epsilon} \in C[-\pi, \pi]$ and a Lebesgue measurable set $F_{\epsilon} \subset[-\pi, \pi]$ such that

$$
\begin{aligned}
\left\|f_{\epsilon}\right\|_{L^{\infty}[-\pi, \pi]} & \leq\|g\|_{L^{\infty}[-\pi, \pi]}, \\
g(\omega) & =f_{\epsilon}(\omega) \quad \text { for all } \omega \in F_{\epsilon},
\end{aligned}
$$

and

$$
\lambda\left([-\pi, \pi] \backslash F_{\epsilon}\right)<\epsilon .
$$

Proof: Lemma 4 is a direct consequence of Lusin's Theorem [27, p. 55].

Equipped with the Lemmas 3 and 4, we are in the position to prove Theorem 7.

Proof of Theorem 7: Let $t \in \mathbb{R}$ be arbitrary but fixed. If we can find a stable LTI system $T_{1}$ such that (17) is not fulfilled the proof is complete, because the assertion then follows directly from Theorem 6.

By Lemma 3 there exists a constant $C_{4}$ such that

$\max _{0 \leq M \leq N}\left(\frac{1}{2 \pi}\right)^{2} \int_{-\pi}^{\pi} \int_{-\pi}^{\pi}\left|\sum_{k=-M}^{M} \mathrm{e}^{i \omega t_{k}} \hat{\phi}_{k}(\eta)\right| \mathrm{d} \omega \mathrm{d} \eta$

$$
\geq C_{4} \log (N)-\frac{1}{C_{L}}
$$

for all $N \in \mathbb{N}$. Therefore, there exists an integer $0 \leq M_{N} \leq N$ such that

$$
\begin{aligned}
\left(\frac{1}{2 \pi}\right)^{2} \int_{-\pi}^{\pi} \int_{-\pi}^{\pi}\left|\sum_{k=-M_{N}}^{M_{N}} \mathrm{e}^{i \omega t_{k}} \hat{\phi}_{k}(\eta)\right| \mathrm{d} \omega \mathrm{d} \eta & \\
& \geq C_{4} \log (N)-\frac{1}{C_{L}}
\end{aligned}
$$

and a $\omega_{N} \in[-\pi, \pi]$ such that

$$
\frac{1}{2 \pi} \int_{-\pi}^{\pi}\left|\sum_{k=-M_{N}}^{M_{N}} \mathrm{e}^{i \omega_{N} t_{k}} \hat{\phi}_{k}(\eta)\right| \mathrm{d} \eta \geq C_{4} \log (N)-\frac{1}{C_{L}} .
$$

For each $N \in \mathbb{N}$, we can choose

$$
\hat{g}_{N}(\eta)=\exp (-i \eta t) \exp \left(i \arg \left(\sum_{k=-M_{N}}^{M_{N}} \mathrm{e}^{i \omega_{N} t_{k}} \hat{\phi}_{k}(\eta)\right)\right) .
$$

Obviously, $\hat{g}_{N} \in L^{\infty}[-\pi, \pi]$ with $\left\|\hat{g}_{N}\right\|_{L^{\infty}[-\pi, \pi]}=1$. Using the abbreviation

$$
\hat{K}_{N}(\eta):=\left(\sum_{k=-M_{N}}^{M_{N}} \mathrm{e}^{i \omega_{N} t_{k}} \hat{\phi}_{k}(\eta)\right) \mathrm{e}^{i \eta t}
$$

we have

$\left|\frac{1}{2 \pi} \int_{-\pi}^{\pi} \hat{K}_{N}(\eta) \hat{g}_{N}(\eta) \mathrm{d} \eta\right|=\frac{1}{2 \pi} \int_{-\pi}^{\pi}\left|\sum_{k=-M_{N}}^{M_{N}} \mathrm{e}^{i \omega_{N} t_{k}} \hat{\phi}_{k}(\eta)\right| \mathrm{d} \eta$

and because of (23),

$$
\left|\frac{1}{2 \pi} \int_{-\pi}^{\pi} \hat{K}_{N}(\eta) \hat{g}_{N}(\eta) \mathrm{d} \eta\right| \geq C_{4} \log (N)-\frac{1}{C_{L}} .
$$

According to Lemma 4, for every $\hat{g}_{N} \in L^{\infty}[-\pi, \pi]$ and every $\epsilon>0$ there exists a function $\hat{h}_{T_{N}, \epsilon} \in C[-\pi, \pi]$ and a set $F_{N, \epsilon} \subset$ $[-\pi, \pi]$ such that

$$
\begin{aligned}
\left\|\hat{h}_{T_{N}, \epsilon}\right\|_{L^{\infty}[-\pi, \pi]} & \leq\left\|\hat{g}_{N}\right\|_{L^{\infty}[-\pi, \pi]} \\
\hat{h}_{T_{N}, \epsilon}(\omega) & =\hat{g}_{N}(\omega) \quad \text { for all } \omega \in F_{N, \epsilon}
\end{aligned}
$$

and

$$
\lambda\left([-\pi, \pi] \backslash F_{N, \epsilon}\right)<\epsilon
$$

Obviously, $\hat{h}_{T_{N}, \epsilon} \in L^{\infty}[-\pi, \pi]$ and therefore every $\hat{h}_{T_{N}, \epsilon}$ defines a stable LTI system $T_{N, \epsilon}$ with norm $\left\|T_{N, \epsilon}\right\| \stackrel{=}{=}$ $\left\|\hat{h}_{T, \epsilon}\right\|_{L^{\infty}[-\pi, \pi]} \leq 1$. Furthermore, we have

$$
\begin{aligned}
& \left|\frac{1}{2 \pi} \int_{-\pi}^{\pi} \hat{K}_{N}(\eta) \hat{h}_{T_{N}, \epsilon}(\eta) \mathrm{d} \eta\right| \\
& \geq\left|\frac{1}{2 \pi} \int_{-\pi}^{\pi} \hat{K}_{N}(\eta) \hat{g}_{N}(\eta) \mathrm{d} \eta\right| \\
& \quad-\left|\frac{1}{2 \pi} \int_{-\pi}^{\pi} \hat{K}_{N}(\eta)\left(\hat{h}_{T_{N}, \epsilon}(\eta)-\hat{g}_{N}(\eta)\right) \mathrm{d} \eta\right| .
\end{aligned}
$$

The second term in (27) can be made arbitrarily small, because

$$
\begin{aligned}
&\left|\frac{1}{2 \pi} \int_{-\pi}^{\pi} \hat{K}_{N}(\eta)\left(\hat{h}_{T_{N}, \epsilon}(\eta)-\hat{g}_{N}(\eta)\right) \mathrm{d} \eta\right| \\
& \leq \frac{1}{2 \pi} \int_{F_{N, \epsilon}}\left|\hat{K}_{N}(\eta)\right|\left|\hat{h}_{T_{N}, \epsilon}(\eta)-\hat{g}_{N}(\eta)\right| \mathrm{d} \eta \\
& \quad+\frac{1}{2 \pi} \int_{[-\pi, \pi] \backslash F_{N, \epsilon}}\left|\hat{K}_{N}(\eta)\right|\left|\hat{h}_{T_{N}}(\eta)-\hat{g}_{N}(\eta)\right| \mathrm{d} \eta \\
& \leq 2\left\|\hat{g}_{N}\right\|_{L^{\infty}[-\pi, \pi]} \frac{1}{2 \pi} \int_{[-\pi, \pi] \backslash F_{N, \epsilon}}\left|\hat{K}_{N}(\eta)\right| \mathrm{d} \eta
\end{aligned}
$$

where we used (25) and (26) in the last line. Since $\lambda([-\pi, \pi] \backslash$ $\left.F_{N, \epsilon}\right)<\epsilon$, we have

$$
\lim _{\epsilon \rightarrow \infty} \int_{[-\pi, \pi] \backslash F_{N, \epsilon}}\left|\hat{K}_{N}(\eta)\right| \mathrm{d} \eta=0
$$

by the absolute continuity of the integral. It follows that

$$
\begin{array}{r}
\sup _{\substack{\hat{h}_{T} \in C[-\pi, \pi] \\
\left\|\hat{h}_{T}\right\|_{L^{\infty}[-\pi, \pi]} \leq 1}}\left|\frac{1}{2 \pi} \int_{-\pi}^{\pi} \hat{K}_{N}(\eta) \hat{h}_{T}(\eta) \mathrm{d} \eta\right| \\
\geq\left|\frac{1}{2 \pi} \int_{-\pi}^{\pi} \hat{K}_{N}(\eta) \hat{g}_{N}(\eta) \mathrm{d} \eta\right| .
\end{array}
$$

Combining (28) with (24) gives

$$
\sup _{\substack{\hat{h}_{T} \in C[-\pi, \pi] \\\left\|\hat{h}_{T}\right\|_{L} \infty[-\pi, \pi] \leq 1}}\left|\frac{1}{2 \pi} \int_{-\pi}^{\pi} \hat{K}_{N}(\eta) \hat{h}_{T}(\eta) \mathrm{d} \eta\right| \geq C_{4} \log (N)-\frac{1}{C_{L}} .
$$

Thus, by the Banach-Steinhaus theorem [27, p. 98], there exists a continuous function $\hat{h}_{T_{1}} \in C[-\pi, \pi]$ such that

$$
\limsup _{N \rightarrow \infty}\left|\frac{1}{2 \pi} \int_{-\pi}^{\pi} \hat{K}_{N}(\eta) \hat{h}_{T_{1}}(\eta) \mathrm{d} \eta\right|=\infty .
$$


Clearly, $h_{T_{1}} \in L^{\infty}[-\pi, \pi]$ and thus $h_{T_{1}}$ defines a stable LTI system $T_{1}$. Furthermore, since

$$
\begin{aligned}
\mid & \sum_{k=-M_{N}}^{M_{N}} \mathrm{e}^{i \omega_{N} t_{k}}\left(T_{1} \phi_{k}\right)(t) \mid \\
& =\left|\frac{1}{2 \pi} \int_{-\pi}^{\pi}\left(\sum_{k=-M_{N}}^{M_{N}} \mathrm{e}^{i \omega_{N} t_{k}} \hat{\phi}_{k}(\eta)\right) \hat{h}_{T_{1}}(\eta) \mathrm{e}^{i \eta t} \mathrm{~d} \eta\right| \\
& =\left|\frac{1}{2 \pi} \int_{-\pi}^{\pi} \hat{K}_{N}(\eta) \hat{h}_{T_{1}}(\eta) \mathrm{d} \eta\right|
\end{aligned}
$$

where we used (1) in the first line, we obtain

$$
\limsup _{N \rightarrow \infty} \max _{|\omega| \leq \pi}\left|\sum_{k=-N}^{N} \mathrm{e}^{i \omega t_{k}}\left(T_{1} \phi_{k}\right)(t)\right|=\infty .
$$

This shows that (17) is not fulfilled and therefore completes the proof.

\section{DiscusSiOn AND OPEN PROBlems}

Although we have not proved Conjecture 1 in this paper, we have shown the divergence of (2) for certain signals in $\mathcal{P} \mathcal{W}_{\pi}^{1}$ and stable LTI system. The sampling-type system approximation process (2) is an important generalization of the ordinary sampling series. For the proof, we used a deep result by Szarek. However, the proof technique seems to not be strong enough to prove Conjecture 1, i.e., the divergence of the sampling series when the system is the identity.

A further open question concerns the convergence behavior of the sampling series (2) with oversampling, i.e., the case where $f \in \mathcal{P W}_{\sigma}^{1}, \sigma<\pi$. It is well known that, in certain situations, oversampling can help resolve convergence problems. For example, the peak value of the Shannon sampling series

$$
\sum_{k=-\infty}^{\infty} f(k) \frac{\sin (\pi(t-k))}{\pi(t-k)}
$$

diverges for certain signals $f \in \mathcal{P W}_{\pi}^{1}$, whereas the series is uniformly convergent for all signals in $f \in \mathcal{P W}_{\sigma}^{1}, \sigma<\pi$, i.e., for the case where oversampling is applied [26]. In contrast, for the system approximation problem with equidistant sampling series, oversampling does not help. In [30] it was shown that there exists a stable LTI system and a signal in $\mathcal{P} \mathcal{W}_{\pi}^{1}$ such that the approximation process diverges. We conjecture that for the system approximation problem with sampling series that use non-equidistant sampling patterns, oversampling does not help either.

Conjecture 2: The divergence of (2), as observed in this paper, remains even if oversampling is applied.

We strongly believe that Conjecture 2 is true. Thus, it is problematic to use the approximation process (2). However, there may be a remedy if the following conjecture is true.

In the approximation process (2), the samples $\left\{f\left(t_{k}\right)\right\}_{k \in \mathbb{Z}}$ of the signal $f$ are used. The sampling of the signal $f$ corresponds to a point evaluation of $f$ at the sampling points $\left\{t_{k}\right\}_{k \in \mathbb{Z}}$. Instead of the point evaluation, it is also possible to consider more general linear functionals $c_{k}: \mathcal{P W} \mathcal{W}_{\pi}^{1} \rightarrow \mathbb{C}, k \in \mathbb{Z}$, for example, functionals that also take the signal values in the proximity of the sampling points $t_{k}, k \in \mathbb{Z}$, into account. In this case, the approximation process takes the form

$$
\sum_{k=-N}^{N} c_{k}(f)\left(T \phi_{k}\right)(t) .
$$

Note that, in the classical sampling approach, the functionals are given by $c_{k}(f)=f\left(t_{k}\right), k \in \mathbb{Z}$.

For approximation processes that use the general evaluation functionals, we have the following conjecture.

Conjecture 3: We conjecture that the system approximation process (29) with properly chosen functionals $c_{k}, k \in \mathbb{Z}$, is uniformly convergent for all stable LTI systems $T$ and all $f \in \mathcal{P W}_{\sigma}^{1}, \sigma<\pi$, i.e., if oversampling is used. However, if no oversampling is used, such more general functionals do not improve the convergence behavior.

Such a positive result would be important for the foundations of digital signal processing, and it would be interesting to find suitable functionals.

\section{APPENDIX}

\section{A. Proof of Lemma 2}

Proof: Let $t \in \mathbb{R}$ and $N \in \mathbb{N}$ arbitrary but fixed. For convenience, we introduce the function

$$
g(\omega):=\sum_{k=-N}^{N} \mathrm{e}^{i \omega t_{k}}\left(T \phi_{k}\right)(t) .
$$

We have

$$
\begin{aligned}
& \left|\sum_{k=-N}^{N} f\left(t_{k}\right)\left(T \phi_{k}\right)(t)\right| \\
& \quad=\left|\frac{1}{2 \pi} \int_{-\pi}^{\pi} \hat{f}(\omega) g(\omega) \mathrm{d} \omega\right| \\
& \quad \leq \frac{1}{2 \pi} \int_{-\pi}^{\pi}|\hat{f}(\omega)||g(\omega)| \mathrm{d} \omega \\
& \quad \leq \max _{|\omega| \leq \pi}|g(\omega)| \frac{1}{2 \pi} \int_{-\pi}^{\pi}|\hat{f}(\omega)| \mathrm{d} \omega \\
& \quad=\max _{|\omega| \leq \pi}|g(\omega)| \mid f \|_{\mathcal{P} \mathcal{W}_{\pi}^{1}}^{1}
\end{aligned}
$$

because $g$ is continuous. Taking the supremum on both sides of (30) gives

$$
\begin{aligned}
\sup _{\|f\|_{\mathcal{P W} 1}^{1} \leq 1}\left|\sum_{k=-N}^{N} f\left(t_{k}\right)\left(T \phi_{k}\right)(t)\right| \\
\leq \max _{|\omega| \leq \pi}\left|\sum_{k=-N}^{N} \mathrm{e}^{i \omega t_{k}}\left(T \phi_{k}\right)(t)\right| .
\end{aligned}
$$

Furthermore, since $g$ is continuous on the compact interval $[-\pi, \pi],|g|$ attains its maximum in some point $\omega^{*} \in[-\pi, \pi]$. For $n \in \mathbb{N}$, let

$$
E_{n}=\left\{\omega:|g(\omega)| \geq\left|g\left(\omega^{*}\right)\right|-\frac{1}{n}\right\}
$$


and choose

$$
f_{n}(t):=\frac{1}{2 \pi} \int_{-\pi}^{\pi} \frac{2 \pi}{\lambda\left(E_{n}\right)} \mathbf{1}_{E_{n}}(\omega) \mathrm{e}^{i \rho(\omega)} \mathrm{e}^{i \omega t} \mathrm{~d} \omega
$$

where $\rho(\omega)=-\arg (g(\omega))$. $\mathbf{1}_{E}$ denotes the indicator function of the set $E$ and $\lambda$ the Lebesgue measure. Obviously, $\left\|f_{n}\right\|_{\mathcal{P} \mathcal{W}_{\pi}^{1}}=1$ for all $n \in \mathbb{N}$. It follows that

$$
\begin{aligned}
& \left|\sum_{k=-N}^{N} f_{n}\left(t_{k}\right)\left(T \phi_{k}\right)(t)\right| \\
& \quad=\left|\frac{1}{2 \pi} \int_{-\pi}^{\pi} \hat{f}_{n}(\omega) g(\omega) \mathrm{d} \omega\right| \\
& \quad=\left|\frac{1}{2 \pi} \int_{-\pi}^{\pi} \frac{2 \pi}{\lambda\left(E_{n}\right)} \mathbf{1}_{E_{n}}(\omega) \mathrm{e}^{i \rho(\omega)} g(\omega) \mathrm{d} \omega\right| \\
& \quad=\frac{1}{\lambda\left(E_{n}\right)} \int_{E_{n}}|g(\omega)| \mathrm{d} \omega \\
& \quad \geq\left|g\left(\omega^{*}\right)\right|-\frac{1}{n},
\end{aligned}
$$

and

$$
\begin{aligned}
& \sup _{\|f\|_{\mathcal{P} \mathcal{N}}^{1} \leq 1}\left|\sum_{k=-N}^{N} f\left(t_{k}\right)\left(T \phi_{k}\right)(t)\right| \\
& \geq \lim _{n \rightarrow \infty}\left|\sum_{k=-N}^{N} f_{n}\left(t_{k}\right)\left(T \phi_{k}\right)(t)\right| \\
& \geq\left|g\left(\omega^{*}\right)\right| \\
& =\max _{|\omega| \leq \pi}\left|\sum_{k=-N}^{N} \mathrm{e}^{i \omega t_{k}}\left(T \phi_{k}\right)(t)\right| .
\end{aligned}
$$

Combining (31) and (32) completes the proof.

\section{B. Proof of Lemma 3}

Proof: We only have to check whether all assumptions of the Proposition in [28, p. 57] are fulfilled. Due to the interpolation property of $\phi_{k}$, we have

$$
\frac{1}{2 \pi} \int_{-\pi}^{\pi} \hat{\phi}_{k}(\omega) \mathrm{e}^{i \omega t_{l}} \mathrm{~d} \omega= \begin{cases}1 & l=k \\ 0 & l \neq k\end{cases}
$$

i.e., $\left\{\hat{g}_{k}, \hat{\phi}_{k}\right\}_{k \in \mathbb{Z}}$, where $\hat{g}_{k}(\omega)=\mathrm{e}^{i \omega t_{k}},|\omega| \leq \pi$, forms a biorthogonal system. In order to be able to apply the Proposition in [28, p. 57], we reorder the functions according to

$$
\begin{aligned}
\left\{\hat{u}_{1}, \hat{u}_{2}, \hat{u}_{3}, \hat{u}_{4}, \ldots\right\} & =\left\{\hat{g}_{0}, \hat{g}_{1}, \hat{g}_{-1}, \hat{g}_{2}, \ldots\right\} \\
\left\{\hat{v}_{1}, \hat{v}_{2}, \hat{v}_{3}, \hat{v}_{4}, \ldots\right\} & =\left\{\hat{\phi}_{0}, \hat{\phi}_{1}, \hat{\phi}_{-1}, \hat{\phi}_{2}, \ldots\right\}
\end{aligned}
$$

which creates a biorthogonal system $\left\{\hat{u}_{k}, \hat{v}_{k}\right\}_{k \in \mathbb{N}}$ with index set $\mathbb{N}$. Obviously, we have $\left\|\hat{u}_{k}\right\|_{\infty}=1$ for all $k \in \mathbb{N}$. Using

$$
f(t)=\sum_{k=1}^{N} a_{k} v_{k}(t)
$$

where $\left\{a_{k}\right\}_{k=1}^{N}$ is an arbitrary sequence of scalars, we obtain

$$
\begin{array}{r}
\frac{1}{2 \pi} \int_{-\pi}^{\pi}\left|\sum_{k=1}^{N} a_{k} \hat{v}_{k}(\omega)\right|^{2} \mathrm{~d} \omega=\|f\|_{\mathcal{P} \mathcal{W}_{\pi}^{2}}^{2} \leq \frac{1}{C_{L}^{2}} \sum_{k=1}^{N}\left|f\left(\tau_{k}\right)\right|^{2} \\
=\frac{1}{C_{L}^{2}} \sum_{k=1}^{N}\left|a_{k}\right|^{2}
\end{array}
$$

where $C_{L}$ is the same constant as in (3) and

$$
\left\{\tau_{1}, \tau_{2}, \tau_{3}, \tau_{4}, \ldots\right\}=\left\{t_{0}, t_{1}, t_{-1}, t_{2}, \ldots\right\}
$$

Thus, all assumptions of the Proposition in [28, p. 57] are fulfilled, and there exists a constant $C_{4}$ such that

$\max _{1 \leq L \leq N}\left(\frac{1}{2 \pi}\right)^{2} \int_{-\pi}^{\pi} \int_{-\pi}^{\pi}\left|\sum_{k=1}^{L} \hat{u}_{k}(\omega) \hat{v}_{k}(\eta)\right| \mathrm{d} \eta \mathrm{d} \omega \geq C_{4} \log (N)$.

Consequently, for every $N$ there exists a natural number $L_{N} \leq$ $N$ such that

$$
\left(\frac{1}{2 \pi}\right)^{2} \int_{-\pi}^{\pi} \int_{-\pi}^{\pi}\left|\sum_{k=1}^{L_{N}} \hat{u}_{k}(\omega) \hat{v}_{k}(\eta)\right| \mathrm{d} \eta \mathrm{d} \omega \geq C_{4} \log (N) .
$$

If $L_{N}$ is odd, then it can be written as $L_{N}=2 M_{N}+1$ with some non-negative integer $M_{N}$, and we have

$$
\begin{aligned}
& \left(\frac{1}{2 \pi}\right)^{2} \int_{-\pi}^{\pi} \int_{-\pi}^{\pi}\left|\sum_{k=-M_{N}}^{M_{N}} \hat{g}_{k}(\omega) \hat{\phi}_{k}(\eta)\right| \mathrm{d} \eta \mathrm{d} \omega \\
& \quad=\left(\frac{1}{2 \pi}\right)^{2} \int_{-\pi}^{\pi} \int_{-\pi}^{\pi}\left|\sum_{k=1}^{L_{N}} \hat{u}_{k}(\omega) \hat{v}_{k}(\eta)\right| \mathrm{d} \eta \mathrm{d} \omega \\
& \geq C_{4} \log (N) .
\end{aligned}
$$

It follows that

$\max _{0 \leq M \leq N}\left(\frac{1}{2 \pi}\right)^{2} \int_{-\pi}^{\pi} \int_{-\pi}^{\pi}\left|\sum_{k=-M}^{M} \hat{g}_{k}(\omega) \hat{\phi}_{k}(\eta)\right| \mathrm{d} \eta \mathrm{d} \omega$ $\geq C_{4} \log (N)$

If $L_{N}$ is even, then we consider $K_{N}=L_{N}-1$ and obtain

$$
\begin{aligned}
& \left(\frac{1}{2 \pi}\right)^{2} \int_{-\pi}^{\pi} \int_{-\pi}^{\pi}\left|\sum_{k=1}^{K_{N}} \hat{u}_{k}(\omega) \hat{v}_{k}(\eta)\right| \mathrm{d} \eta \mathrm{d} \omega \\
& \geq\left(\frac{1}{2 \pi}\right)^{2} \int_{-\pi}^{\pi} \int_{-\pi}^{\pi}\left|\sum_{k=1}^{L_{N}} \hat{u}_{k}(\omega) \hat{v}_{k}(\eta)\right| \mathrm{d} \eta \mathrm{d} \omega \\
& \quad-\left(\frac{1}{2 \pi}\right)^{2} \int_{-\pi}^{\pi} \int_{-\pi}^{\pi}\left|\hat{u}_{L_{N}}(\omega) \hat{v}_{L_{N}}(\eta)\right| \mathrm{d} \eta \mathrm{d} \omega \\
& \geq C_{4} \log (N)-\frac{1}{C_{L}} .
\end{aligned}
$$

Again, $K_{N}$ can be written as $K_{N}=2 M_{N}+1$ with some nonnegative integer $M_{N}$, and we have

$$
\begin{aligned}
& \left(\frac{1}{2 \pi}\right)^{2} \int_{-\pi}^{\pi} \int_{-\pi}^{\pi}\left|\sum_{k=-M_{N}}^{M_{N}} \hat{g}_{k}(\omega) \hat{\phi}_{k}(\eta)\right| \mathrm{d} \eta \mathrm{d} \omega \\
& \quad=\left(\frac{1}{2 \pi}\right)^{2} \int_{-\pi}^{\pi} \int_{-\pi}^{\pi}\left|\sum_{k=1}^{K_{N}} \hat{u}_{k}(\omega) \hat{v}_{k}(\eta)\right| \mathrm{d} \eta \mathrm{d} \omega \\
& \geq C_{4} \log (N)-\frac{1}{C_{L}} .
\end{aligned}
$$


It follows that

$$
\begin{aligned}
\max _{0 \leq M \leq N}\left(\frac{1}{2 \pi}\right)^{2} \int_{-\pi}^{\pi} \int_{-\pi}^{\pi}\left|\sum_{k=-M}^{M} \hat{g}_{k}(\omega) \hat{\phi}_{k}(\eta)\right| \mathrm{d} \eta \mathrm{d} \omega & \\
& \geq C_{4} \log (N)-\frac{1}{C_{L}} .
\end{aligned}
$$

Combining the results for odd and even $M_{N}$ completes the proof.

\section{REFERENCES}

[1] C. E. Shannon, "Communication in the presence of noise," Proc. IRE, vol. 37, no. 1, pp. 10-21, Jan. 1949.

[2] R. P. Feynman, Feynman Lectures on Computation, A. J. Hey and R. W. Allen, Eds. Baltimore, MD: Penguin, 1999.

[3] A. J. Jerri, "The Shannon sampling theorem-its various extensions and applications: A tutorial review," Proc. IEEE, vol. 65, pp. 1565-1596, Nov. 1977.

[4] J. R. Higgins, "Five short stories about the cardinal series," Bull. Amer. Math. Soc., vol. 12, no. 1, pp. 45-89, 1985.

[5] Nonuniform Sampling: Theory and Practice, F. Marvasti, Ed. Boston, MA: Kluwer/Plenum, 2001.

[6] P. L. Butzer and R. L. Stens, "Sampling theory for not necessarily bandlimited functions: A historical overview," SIAM Rev., vol. 34, no. 1, pp. 40-53, Mar. 1992.

[7] P. J. S. G. Ferreira, "Nonuniform sampling of nonbandlimited signals," IEEE Signal Process. Lett., vol. 2, pp. 89-91, May 1995.

[8] J. R. Higgins, Sampling Theory in Fourier and Signal Analysis-Foundations. New York: Oxford Univ. Press, 1996.

[9] P. L. Butzer, W. Splettstößer, and R. L. Stens, "The sampling theorem and linear prediction in signal analysis," Jahresbericht der Deutschen Mathematiker-Vereinigung, vol. 90, no. 1, pp. 1-70, Jan. 1988.

[10] M. K. Habib, "Digital representations of operators on band-limited random signals," IEEE Trans. Inf. Theory, vol. 47, pp. 173-177, Jan. 2001.

[11] M. Zakai, "Band-limited functions and the sampling theorem," Inf. Control, vol. 8, no. 2, pp. 143-158, 1965.

[12] I. Bar-David, "An implicit sampling theorem for bounded bandlimited functions," Inf. Control, vol. 24, no. 1, pp. 36-44, Jan. 1974.

[13] E. Masry and S. Cambanis, "Consistent estimation of continuous-time signals from nonlinear transformations of noisy samples," IEEE Trans. Inf. Theory, vol. 27, no. 1, pp. 84-96, Jan. 1981.

[14] K. Piwnicki, "Modulation methods related to sine-wave crossings," IEEE Trans. Commun., vol. 31, pp. 503-508, Apr. 1983.

[15] R. Duffin and A. C. Schaeffer, "Some properties of functions of exponential type," Bull. Amer. Math. Soc., vol. 44, no. 4, pp. 236-240, Apr. 1938.

[16] H. Boche and U. J. Mönich, "Approximation of wide-sense stationary stochastic processes by Shannon sampling series," IEEE Trans. Inf. Theory, vol. 56, pp. 6459-6469, Dec. 2010.

[17] H. Boche and U. J. Mönich, "General behavior of sampling-based signal and system representation," in Proc. 2008 IEEE Int. Symp. Inf. Theory, Jul. 2008, pp. 2439-2443.

[18] H. Boche and U. J. Mönich, "Non-uniform sampling-Signal and system representation," in Proc. 2008 Int. Symp. Inf. Theory Appl. (ISITA), Auckland, New Zealand, Dec. 2008, pp. 1576-1581.

[19] B. Y. Levin, Lectures on Entire Functions. Providence, RI: Amer. Math. Soc., 1996

[20] R. M. Young, An Introduction to Nonharmonic Fourier Series. New York: Academic, 1980.

[21] Y. I. Lyubarskii and K. Seip, "Complete interpolating sequences for Paley-Wiener spaces and Muckenhoupt's $\left(A_{p}\right)$ condition," Revista Matemática Iberoamericana, vol. 13, no. 2, pp. 361-376, 1997.

[22] R. P. Boas, Entire Functions. New York: Academic, 1954.

[23] R. M. Young, An Introduction to Nonharmonic Fourier Series. New York: Academic, 2001.
[24] H. Boche and U. J. Mönich, "Local and global convergence behavior of non-equidistant sampling series," in Proc. IEEE Int. Conf. Acoust., Speech, Signal Process. (ICASSP), Apr. 2009, pp. 2945-2948.

[25] H. Boche and U. J. Mönich, "Convergence behavior of non-equidistant sampling series," Signal Process., vol. 90, no. 1, pp. 145-156, Jan. 2010.

[26] H. Boche and U. J. Mönich, "Global and local approximation behavior of reconstruction processes for Paley-Wiener functions," Sampling Theory Signal Image Process., vol. 8, no. 1, pp. 23-51, Jan. 2009.

[27] W. Rudin, Real and Complex Analysis, 3rd ed. New York: McGrawHill, 1987.

[28] S. J. Szarek, "Nonexistence of Besselian basis in $C(S)$," J. Funct Anal., vol. 37, pp. 56-67, 1980.

[29] P. du Bois-Reymond, "Nachrichten der Königlichen Gesellschaft der Wissenschaften und der Georg August Universität zu Göttingen," Ueber die Fourierschen Reihen, no. 21, pp. 571-584, Aug. 6, 1873 [Online]. Available: http://gdz.sub.uni-goettingen.de/en/dms/load/ img/?PPN=PPN252457072_1873\&DMDID=dmdlog85

[30] H. Boche and U. J. Mönich, "Sampling-type representations of signals and systems," Sampling Theory Signal Image Process., 2010, accepted for publication.

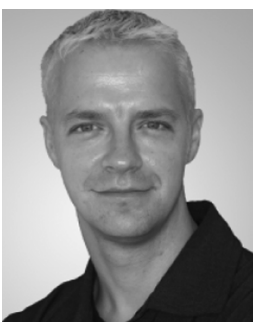

Holger Boche (M'04-SM'07-F'11) received the Dipl.-Ing. and Dr.-Ing. degrees in electrical engineering from the Technische Universität Dresden, Dresden, Germany, in 1990 and 1994, respectively. He graduated in mathematics from the Technische Universität Dresden in 1992, and from 1994 to 1997, he did postgraduate studies in mathematics at the Friedrich-Schiller Universität Jena, Jena, Germany. He received the Dr.rer.nat. degree in pure mathematics from the Technische Universität Berlin, Berlin, Germany, in 1998.

In 1997, he joined the Heinrich-Hertz-Institut (HHI) für Nachrichtentechnik Berlin, Berlin, Germany. Since 2002, he has been a Full Professor for mobile communication networks with the Institute for Communications Systems, Technische Universität Berlin. In 2003, he became Director of the Fraunhofer German-Sino Lab for Mobile Communications, Berlin, Germany and since 2004 he has also been Director of the Fraunhofer Institute for Telecommunications (HHI), Berlin, Germany. Since October 2010, he has been with the Institute of Theoretical Information Technology and Full Professor at the Technische Universität München, Munich, Germany. He was a Visiting Professor with the ETH Zurich, Zurich, Switzerland, during the 2004 and 2006 winter terms and with KTH Stockholm, Stockholm, Sweden, during the 2005 summer term.

Prof. Boche is a Member of IEEE Signal Processing Society SPCOM and SPTM Technical Committee. He was elected a Member of the German Academy of Sciences (Leopoldina) in 2008 and of the Berlin Brandenburg Academy of Sciences and Humanities in 2009. He received the Research Award Technische Kommunikation from the Alcatel SEL Foundation in October 2003, the Innovation Award from the Vodafone Foundation in June 2006 and the Gottfried Wilhelm Leibniz Prize from the Deutsche Forschungsgemeinschaft (German Research Foundation) in 2008. He was corecipient of the 2006 IEEE Signal Processing Society Best Paper Award and recipient of the 2007 IEEE Signal Processing Society Best Paper Award.

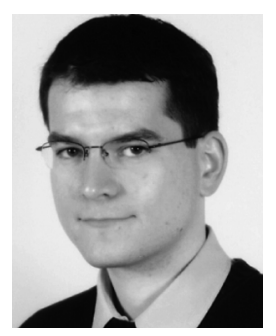

Ullrich J. Mönich (S'06) received the Dipl.-Ing. degree in electrical engineering from the Technische Universität Berlin, Berlin, Germany, in 2005.

During winter term 2003, he was a visiting researcher at the University of California, Santa Barbara. He is currently with the Technische Universität München, Munich, Germany, where he is working towards the Ph.D. degree. His research activities comprise sampling theory, signal processing, and information theory. 